\title{
Index
}

Page numbers in italics refer to figures.

indicates video clip on the accompanying DVD

Abducent nerve (CN VI) 13, 26 lesions 187

Abyssian cat, myasthenia gravis 508

Accessory nerve (CN XI) 16, 26

Acepromazine 414, 418, 419

Acetylcholine 32

Actinomyces spp. 266

Active muscle contraction 492

Acupuncture 496-500

Acute phase proteins 58

Acute renal failure 344

Acute retinal degeneration 177

Acute tubular necrosis 367

Adrenal insufficiency 354

Adverse drug reactions in CNS 467-9 (see also individual drugs)

Afferent nervous system 59

Afghan Hound myelopathy 509 spinal cord disease 276

Airedale Terrier, cerebellar degeneration 509

Akita

cerebellar degeneration 509

congenital vestibular disease 509

glycogenosis 518

myasthenia gravis 509

Alaskan Husky, necrotizing encephalopathy 509

Alaskan Malamute peripheral neuropathy 290, 509

Albino animals, abnormal visual pathways 187

Alfaxalone 415, 419

Alkylating agents 465-6

Allodynia 252

American Bulldog, neuronal ceroid lipofuscinosis 518

American Staffordshire Terrier, ceroid lipofuscinosis 518

Amino acid analysis 347

Amoxicillin 268

Amphotericin B 457-8

Anaesthesia

fluid therapy 429-31

in intracranial disease

induction 414-15

maintenance $415-16$

monitoring 416-17

premedication/sedation 413-14

recovery $417-18$

stabilization $412-13$

supportive care 416

IPPV 423-5 monitoring

blood gas analysis $426-7$

blood pressure 427-8

body temperature 428-9

capnography 426

ECG 427

pulse oximetry $425-6$

neurosurgery 434

in peripheral nervous system disease $422-3$

in spinal disease $418-21$

Analgesia, perioperative 421, 423, 428-9

Anaplasma phagocytophilum 40, 45

Anisocoria 141, 167, 181, 184

Anosmia 214

Antibacterial drugs 455-7

(see also specific drugs)

Antibiotics, adverse CNS reactions 467-8

Anticancer drugs 465-7

(see also specific drugs)

Anticonvulsants

serum concentration $40-1$

toxicity 301,325

Antiepileptic drugs see Anticonvulsants

Antifungal drugs 457-9

(see also specific drugs)

Anti-inflammatory drugs 460-5

(see also specific drugs)

Antiprotozoal drugs 460

(see also specific drugs)

Aortic thrombosis 326-7

Appetite regulation 27

Arachnoid cysts see Intra-arachnoid cysts

Arterial thromboembolism 341

Ascending reticular activating system 4, 28

Aspergillus spp. 45, 266, 268, 457

Aspiration 422

Aspiration pneumonia 486-7

Astrocytoma 299

Ataxia 7

hereditary 514

hypernatraemic 150

neonatal 242, 511

Ataxic breathing 137

Ataxic gait 297-8

Atlantoaxial instability 256, 259, 275, 281-4

Atlantoaxial subluxation 79

Atlanto-occipital overlapping 259, 275

Australian Cattle Dog

ceroid lipofuscinosis 518

polioencephalomyelopathy 509

Australian Kelpie

cerebellar cortical degeneration 509

globoid cell leucodystrophy 518 
Automotor seizures 119

Autonomic control 27

Autoregulation 409

Avermectin toxicity 160-1

Aversion syndrome 5

Awareness 136

Axillary nerve 331

Axonopathy 514

Axonotmesis 330

Azathioprine 211, 266, 463, 469

Babesia spp. 45

Bacterial encephalitis 206-7

Balinese cat, Niemann-Pick type A 517

Barbiturates 401, 407 (see also specific drugs)

Bartonella spp. 45

Basset Hound

cervical spondylomyelopathy 509

Lafora disease 519

methylmalonic aciduria 519

Beagle

cerebellar cortical degeneration 509

congenital vestibular disease 509

globoid cell leucodystrophy 519

GM1 gangliosidosis 519

idiopathic epilepsy 509

Lafora disease 519

spinal cord disease 276

steroid-responsive meningitis 55, 509

Bedding 420-1, 484

Behaviour

changes in, lesion localization 27, 138

emotional 27

examination 4,5

limbic system 140

perception and cognition 138

rehabilitation 487-8

Belgian Tervueren

epilepsy 119, 509

muscular dystrophy 509

Bengal cat, demyelinating polyneuropathy 291

Benzodiazepines 125, 126, 131, 133, 405, 407, 413, 415,419

(see also specific drugs)

Bernese Mountain Dog

dysmyelination 510

hepatocerebellar disease 510

steroid-responsive meningitis-arteritis 55,510

Bethanecol 386

Biceps brachii reflex 20

Biopsy

brain $101-6,447-8$

muscle $94-8,348$

nerve 348

peripheral nerve $98-100$

sample submission 93

Biot's breathing 137

Birman cat

abnormal visual pathways 187,188

distal central-peripheral axonopathy 508

distal polyneuropathy 291

spongiform encephalopathy 508

Bladder and bowel management 482-4

Blastomyces dermatitidis 45

Blastomycosis 158

Blepharospasm 167

Blindness 149, 175, 178, 179, 180, 181 lesion localization 27

(see also Vision, disorders)

Block vertebrae 79, 312

Blood gases 396

Blood glucose 396

Blood lead concentration 43

Blood pressure monitoring 396, 427-8

Blood transfusion 420

Body temperature

monitoring 396, 428

regulation 27

Border Collie

cerebellar cortical degeneration 510

ceroid lipofuscinosis 519

deafness 510

idiopathic epilepsy 510

methylmalonic aciduria 519

peripheral sensory neuropathy 510

sensory neuropathy 290

Borrelia burgdorferi 45

Borzoi, Wobbler syndrome 510

Boston Terrier

divergent strabismus 188

glioma 152

hemivertebrae 510

hydrocephalus 145,510

Botulism 227, 290, 295

Bouvier des Flandres idiopathic laryngeal paralysis 510 myopathy 510

Boxer

degenerative myelopathy 510

giant axonal neuropathy 291

glioma 152

immune-mediated polymyositis 510

Niemann-Pick type C 519

progressive axonopathy 290, 510

spinal cord disease 276

steroid-responsive meningitis 55,510

Brachial plexus 25

avulsion 337-8

Brachiocephalic nerve 331

Bradycardia 137

Brain

anatomy 25, 26

biopsy 101-6, 447-8

cysts 204

herniation 140

injury 433

tumours, 151-8, 198, 204

radiation therapy $472-5$

Brain-heart syndrome 137

Brainstem 25, 27-8

auditory evoked potentials 60, 69-72, 224

Brittany Spaniel

cerebellar degeneration 510

motor neuron disease 290, 510

Brivacetam 126

Bromide 125, 126, 128

Brucella canis 45, 266, 268

Brussels Griffon

Chiari-like malformation 510

syringomyelia 510

Bull Terrier

cerebellar degeneration 511

idiopathic laryngeal paralysis 511

Bullmastiff, spongiosis of grey matter 511

Buprenorphine 429 
Burmese cat

congenital vestibular disease 508

GM2 gangliosidosis 517

hypokalaemia 508

Butorphanol 413, 415, 429

Butterfly vertebrae $79,312,375$

Cairn Terrier

cerebellar degeneration 511

globoid cell leucodystrophy 290, 519

hydrocephalus 145

multisystem neuronal degeneration 143, 511

spinal cord disease 276

Calcinosis circumscripta 82, 256, 259, 275, 281, 299, 301, 311

Calvarial fractures 402

Candida spp. 457

Canine distemper viral myelitis 320-1

Canine distemper virus $38,40,45,207-8$

Canine dysautonomia 378

Capnography 426

Carbamate toxicity $41,184,352$

Carbamazepine 126

Carbapenems 456

Cardiovascular disease 177

Cardiovascular system, regulation 28

Carisbamate 126

Carmustine 466

L-Carnitine 360

Carnitine analysis 347

Carprofen 429

Cataplexy 8, 160, 250 lesion localization 27

Cauda equina 369

Caudal cutaneous antebrachial nerve 331

Caudal cutaneous sural nerve 331

Caudal fossa craniotomy 444

Caudal lumbar vertebrae trauma 339

Cavalier King Charles Spaniel Chiari-like malformations 511 hypertonicity 249,511

syringomyelia 511

Cavernous sinus syndrome 184-5, 189

Cefalexin 268

Cefazolin 268

Cefotaxime 455,469

Cefpodoxime proxetil 456

Ceftazidime 456, 469

Ceftiofur 456

Central afferent pathways $67-8$

Central axonopathies 299

Central lipofuscinosis 240

Central neurogenic hyperventilation 137

Central vestibular diseases 199, 203-12

Centronuclear myopathy 357-8

Cephalosporins 455-6 (see also specific drugs)

Cerebellar ataxia, neurological examination

Cerebellar cortical abiotrophy $508509,512,515,516$

Cerebellar cortical degeneration 239, 509, 510, 511, 513,514

Cerebellar degeneration 509, 510, 511, 512

Cerebellar disease 185, 239-43

Cerebellar dysfunction 234-5

Cerebellar dysplasia 514

Cerebellar hypoplasia 511, 517

Cerebellum 25, 26, 28

Cerebral blood flow 409-10
Cerebral cortex 4, 60

Cerebral dysfunction 235-6

Cerebral perfusion pressure 410-11

Cerebrospinal fluid (CSF)

acute phase proteins 58

collection $46-8$

cytology $49,51-8$

electrophoresis 58

flow study

microprotein 51, 53

PCR 58

physical characteristics $50-1$

production 45-6

sample handling 48-50

Cerebrovascular disease 198, 212

Cerebrum 25, 27

Ceroid lipofuscinosis 518, 519, 520, 521

Cervical disc disease, acute $279-80$

Cervical disc fenestration 440

Cervical spondylomyelopathy 509

Cervical stenotic myelopathy see Wobbler syndrome

Cervical vertebral column stabilization $439-50$

Cervical vertebral column surgery

cervical disc fenestration 440

cervical vertebral column stabilization $439-40$

dorsal laminectomy 437-8

lateral laminectomy $438-9$

ventral decompression (ventral slot) 436-7

Cervicothoracic intumescence 25

Chagas' disease 363

Channelopathies 109-10

Chemoreceptor trigger zone 452

Chesapeake Bay Retriever

degenerative myelopathy 511

distal sensorimotor polyneuropathy 290

spinal cord disease 276

steroid-responsive meningitis-arteritis 511

Cheyne Stokes respiration 137

Chiari-like malformations

breed associations 510, 511

MRI 147, 260

and spinal pain 256

and syringomyelia $260-3$

and tetraparesis 275,285

and vestibular disease 197, 203

Chihuahua

ceroid lipofuscinosis 519

hydrocephalus 145,511

necrotizing meningoencephalitis 54, 511

Chinese herbal medicines 500

Chinese veterinary medicine acupoints and channels 496-500

evolution 500-1

herbal medicines 500

pattern diagnoses 500

treatment of neurological disorders $502-7$

Chinooks, paroxysmal dyskinesia 249-50

Chloramphenicol 456, 469

Cholinesterase 41

Chordoma 370, 376-7

Choroid plexus tumours 152, 153

Chow Chow

cerebellar hypoplasia 511

dysmyelination 511

myotonia congenital 511

Chromatic pupillary light reflex 170

Chronic inflammatory demyelinating polyneuropathy 290, 293 
Ciclosporin 211, 464

Circling 8 lesion localization 27

Cisapride 386

Clindamycin 460, 469

Clinical pathology

anticonvulsant serum concentration $40-1$

bile acids and ammonia 41

blood lead concentration 43

cerebrospinal fluid $45-58$

cholinesterase 41

congenital disease testing $41-2$

endocrine testing 42

haemostasis $42-3$

markers of muscular injury 43-4

serology and microbiology 44-5

serum osmolality and osmolal gap 44

serum protein electrophoresis 44

standard minimum database $36-40$

urine protein:creatinine ratio 44

Clonazepam 133

Clorazepate 128, 131

Clostridium 362 tetani 456

Clumber Spaniel, pyruvate dehydrogenase deficiency 519

Cluster seizures 403 (see also Status epilepticus)

Coccidioides immitis 45, 457

Coccidioidomycosis 158

Coccygeal muscle injury 380-1

Cocker Spaniel,

ceroid lipofuscinosis 519

congenital vestibular disease 511

neuronal degeneration 143,511

Coenzyme Q10 360

Cognition 136, 138

Cognitive disorder 144-5

Collapse 342, 345, 512, 514

(see also Exercise intolerance; Seizures)

Collies

cerebellar degeneration 511

dermatomyositis 511

Coma

neuroaxonal dystrophy 511

aetiology 141

avermectin toxicity 160

brain tumours 151

clinical signs 137

diagnosis 141-2

hypoglycaemic 149

hypothyroid 151

ivermectin toxicity 160

lesion localization 27, 138

management 143

monitoring 142-3

prognosis 143

Common peroneal (fibular) nerve 331

Complex repetitive discharges 62

Compound action potentials 60

Computed tomography $85-7,332$

Conscious proprioception 17

Consciousness 4, 138

altered $136-7$

regulation 27,28

Continuous dorsal laminectomy 435

Contusion 388

Core-like myopathy 513
Corneal reflex 9, 10, 172, 187, 192

Corticosteroids

adverse reactions 462

CNS infections 460-1

head injury 400

immunosuppression 461-2

spinal trauma 461

(see also specific drugs)

Coton de Tulear

cerebellar degeneration 511

degeneration 243

immune-mediated cerebellar granuloprival neonatal ataxia 242, 511

Coupage 487

Cranial nerves 26, 27, 213

abducent (CN VI) 13, 26

abnormalities

brainstem lesions 28

cerebellar lesions 28

forebrain lesions 27

muscle lesions 33

peripheral nerve lesions 32

spinal cord lesions 30-1

accessory $(\mathrm{CN} \mathrm{XI})$ 16, 26

assessment 9-16

deficit 222, 230

facial (CN VII) 13-14, 26, 217

glossopharyngeal (CN IX) 16, 26

H-reflex 65, 66

hypoglossal (CN XII) 16, 26

iatrogenic lesions 230

oculomotor (CN III) 10-11, 26

olfactory (CN I) 9, 26, 27

optic (CN II) 9-10, 26

trigeminal (CN V) 12-13, 26, 214, 215, 216

trochlear (CN IV) 11-12, 26

vagus $(C N X) 16,26$

vestibulocochlear (CN VIII) 14-16, 26

Cranial surgery

complications 449-50

indications $444-5$

neuroendoscopy 447

patient positioning 445

rostrotentorial craniotomy 446

suboccipital craniotomy 447

transfrontal craniotomy 445

transsphenoidal hypophysectomy 447

transverse sinus occlusion 446

Cranial tibial reflex 21-2

Craniectomy 102, 103-4, 444

Craniotomy 444

Creatine kinase 43, 346

Cretan Hound, spongiform leucoencephalopathy 511

'Crocodile tears' 194

Cryotherapy 488,490

Cryptococcosis 45, 158, 210 meningitis 56

CSF see Cerebrospinal fluid

Curly Coated Retriever exercise-induced collapse 512 glycogen storage disease 519

Cushing's myopathy 353-4

Cutaneous trunci (panniculus) reflex 23

Cytarabine 211, 466-7, 469

Cytochrome oxidase deficiency 520

Dachshund ceroid lipofuscinosis 519 


\section{Dachshund continued}

congenital myasthenia gravis 512

idiopathic epilepsy 512

Lafora disease 519

mucopolysaccharidosis 519

narcolepsy-cataplexy 512

sensory neuropathy 512

Dalmatian

cerebral and spinal leucodystrophy 512

ceroid lipofuscinosis 519

congenital deafness 512

laryngeal paralysis-polyneuropathy complex 290, 512

spinal cord disease 276

spongiform degeneration 143

DAMNITV classification 33-5, 523-4

Dantrolene 387

Dazzle reflex 171

Deafness 216-17

brainstem auditory evoked potentials 60, 69-72, 224

breed associations 510, 512

diagnosis 225

differential diagnosis 222

lesion localization 27

pathogenesis 225

treatment and prognosis 225

Decerebellate rigidity 6

Decerebrate rigidity $6,27,137$

Decompression 436

Decubital ulcers 485-6

Deep dorsal laminectomy 435

Deep peroneal (fibular) nerve 331

Degenerative lumbosacral stenosis $370-4$

Degenerative myelopathy $275,299,301,302-3$ breed associations 513, 515, 516

Dehydration 422

Delirium 136 lesion localization 27

Dementia 136 lesion localization 27

Depression 136 lesion localization 27

Dermatomyositis $364,511,516$

Dermoid sinus 256, 275, 285, 301, 314

Desflurane 416

Detrusor muscle, innervation 381

Detrusor reflex 382

Devon Rex cat, hereditary myopathy 508

Dexamethasone 469

Dexmedetomidine 414, 419

Dextran 40399

Dextran 70399

Diabetes mellitus 36, 290, 292, 301

Diabetic hyperosmolar non-ketotic syndrome 44

Diazepam 131, 133, 387, 414, 415, 419

Diencephalon 27

Diethylstilboestrol 387

Diffuse idiopathic skeletal hyperostosis 299, 301, 322, 323, 370

Dirofilaria immitis 45

Disc disease 81

Discal cysts 275, 280, 281

Discography 83

Discospondylitis/osteomyelitis 80,301 clinical signs 256, 266, 275, 285, 299, 320, 370, 377 diagnosis

clinical pathology $38,266-7,268$ imaging 267-8

pathogenesis 266

prognosis 269

treatment 268-9

Distal central-peripheral axonopathy 508

Distal denervating disease 290, 292

Distal myopathy 516

Distal symmetrical polyneuropathy 513

Distemper 158

Diuretics 413

DNA testing 114-16

Dobermann congenital vesitibular disease and deafness 512 'dancing Dobermann disease' 250, 512 motor neuron disease 290 narcolepsy-cataplexy 512 Wobbler syndrome 512

Domestic Longhaired cat, hyperchylomicronaemia 291

Domestic Shorthaired cat cerebellar abiotrophy 508 hyperchylomicronaemia 291 hyperoxaluria 291 neuroaxonal dystrophy 508 X-linked muscular dystrophy 508

Doppler ultrasonography 92

Dorsal funiculus 28

Dorsal laminectomy 435, 437-8, 441, 443

Dorsal metencephalon see Cerebellum

Doxycycline 268

Dropped jaw 13, 215, 229 differential diagnosis 222 lesion localization 27

Drug therapy adverse reactions $290,295,467-9$

anticancer drugs $465-7$ anti-infectious drugs $455-60$ anti-inflammatory therapy $460-5$ blood-brain barrier 452-3 drug entry into brain 452-4 (see also individual drugs)

Dural ossification 258-9, 299, 301, 303

Dysautonomia 185, 227, 370

Dyskinesia 233, 234, 236 forebrain lesions 27

Dysmetria 7

Dysmyelination 244, 510, 511, 516

Dysphagia 217-19 differential diagnosis 222 lesion localization 27

Dysphonia 16, 219

Dyssynergia 386

Dysthesia 252

Dystroglycan deficiency 357

Dystrophin deficiency 355-6

Ear, tumours 199-200 (see also Otitis)

ECG see Electrocardiography

EEG see Electroencephalography

Efferent nervous system 59-60

Effleurage 491

Egyptian Mau cat, spongiform encephalopathy 143, 508

Ehrlichia canis 362

Ehrlichiosis 38, 45, 54, 158

Electrocardiography, anaesthetic monitoring 427

Electroencephalography 72-6, 408

Electromyography $61-2,64-5,238,332,370,423$ 
Electrophysiology 332, 348

afferent nervous system 59

central afferent pathways $67-8$

cerebral cortex 60

compound action potentials 60

efferent nervous system $59-60$

electroencephalography $72-3$

field potentials 60

peripheral motor system $61-5$

peripheral sensory system $65-7$

special senses $60,69-72$

Electroretinography 174, 175

Emergencies

head trauma 394-403

seizures 134-5

spinal cord injury 388-94

status epilepticus and cluster seizures 403-8 surgical $432-3$

EMG see Electromyography

Empyema 256, 269, 275, 285-6, 301

Encephalitis 158

Encephalitozoon cuniculi 45

Encephalopathy

feline ischaemic 166

hypertensive 165-6

Endocrine myopathy 353-5

Endocrine neuropathy 292, 316

Endocrine testing 42

English Bulldog

cerebellar cortical degeneration 512

divergent strabismus 188

hemivertebrae 512

hydrocephalus 145

spina bifida 512

English Pointer

motor neuron disease 290, 512

sensory neuropathy 290, 512

English Setter, ceroid lipofuscinosis 519

English Spaniel, sensory neuropathy 290

English Springer Spaniel

congenital myasthenia gravis 516

dysmelination 516

fucosidosis 520

GM1 gangliosidosis 520

Enilconazole 458

Enophthalmos 167, 182, 192

Enrofloxacin 268

Enterobacteriaceae 456

Eosinophilic pleocytosis 55-6

Ependymoma 152, 299, 301, 370

Epidural haemorrhage 275, 299, 301

Epidurography 83, 370

Epilepsia partialis continua 118

Epilepsy see Seizures

Episodic hypertonicity 511

Episodic weakness see Exercise intolerance

Escherichia coli 266, 268, 455

Esotropia 167, 188, 190

Ethylene glycol toxicity 39

European cat, hyperchylomicronaemia 291

Evoked injury potential 68

Exercise intolerance

clinical signs 342

differential diagnosis 345

disorders of neuromuscular transmission 349-52

lesion localization 342, 343

myopathies 352-67

neurodiagnostic investigation 345 pathophysiology 342, 344

peripheral neuropathies 349

Exophthalmos 167

Exotropia 167, 186, 187, 188

Extensor carpi radialis reflex 20

Extensor postural thrusting

External ophthalmoplegia 214-15

Extracranial structures 25

Extraocular muscle evaluation 171

Extraocular myositis 189-90

Extrapyramidal system 27

Eye

diseases see Vision and individual diseases sympathetic innervation 173, 220

Eyeball

position and movement,

assessment 171-2

disorders 175, 186-91

somatosensory innervation 172

Eyelid

abnormalities $175,191-3$

motor control 173

opening disorders 192

paralysis, lesion localization 27

somatosensory innervation 172

third see Third eyelid

F waves 63-4

Face

abnormalities 213-31

sympathetic innervation 173

(see also Facial)

Facetectomy 435, 443

Facial asymmetry 14

Facial expression abnormalities 216, 217

Facial hyperaesthesia

Facial hypoalgesia in forebrain lesions 27

Facial nerve (CN VII) 13-14, 26, 217

Facial paralysis 229-30

differential diagnosis 222

Faecal incontinence 31

Falling 27

Familial reflex myoclonus 514

Fasciculations 234, 236

Felbamate 125, 126, 128, 131

Feline cerebellar hypoplasia 242

Feline coronavirus 208-9

Feline dysautonomia $377-8$

Feline encephalomyelitis 246

Feline encephalomyelopathy 244

Feline hyperaesthesia syndrome 247-8

Feline immunodeficiency virus $38,45,362$

Feline infectious meningitis 321

Feline infectious myelitis 321

Feline infectious peritonitis 38, 45, 158, 208-9, 321 (see also Feline coronavirus)

Feline intervertebral disc disease 310

Feline ischaemic encephalopathy 166

Feline leukaemia 38, 45

Feline leukaemia virus-associated myelopathy 321

Feline spongiform encephalopathy 246

Femoral nerve 330, 331 injury 341

Fenestration 435, 440, 442

Fentanyl 413, 415, 419, 428

Fibrocartilaginous embolism 275, 289-90, 299, 301, 325-6, 341, 370, 381

Fibrosing esotropia 190 
Fibrotic myopathy 365-6, 513

Field potentials 60

Fine needle aspiration 57

Finnish Harrier, cerebellar degeneration 512

Flaccidity 298

Flow-metabolism coupling 409-10

Fluconazole 268, 457, 459, 469

Flucytosine 459

Fluid therapy 399, 429-31

Flurofelbamate 126

Fluoroquinolones 457 and acute retinal degeneration 177

Foramenotomy 435, 443

Foraminal stenosis $332-4$

Forebrain 27

Fosphenytoin 407

Fox Hound, spinal cord disease 276

French Bulldog, hemivertebrae 512

French Spaniel, sensory neuropathy 290

Friction massage 491

Frontal lobe 26

Fructosamine 42

Fucosidosis 240, 520

Functional neuroanatomy 25-7

GABA receptors 125

Gabapentin 125, 126, 128, 131, 133, 134, 429

Gag reflex 9

Gait

abnormalities 297-8

lesion localization 27-8, 30-33, 301-2

evaluation $6-8$

training 493-5

Galactosialidosis 240

Gammel Dansk Honschund, congenital myasthenia gravis 512

Ganglioradiculitis 290, 293

Gastrocnemius reflex 21-2

Gemistocytic astrocytoma 106

General physical examination 4

Generalized tonic-clonic seizure

Generalized tremor syndrome of dogs 245-6

Genetic diseases

congenital 108

channelopathies 109-10

counselling 115-16

DNA tests $114-16$

gene discovery strategies $112-14$

neurodegenerative 110

inborn errors of metabolism 108-9

recognition 107-10

segregation analysis 110-12

Geriatric tremors 246

German Shepherd Dog

congenital vestibular disease 512

degenerative myelopathy 513

fibrotic myopathy 512

giant axonal neuropathy 291, 513

glycogenosis 520

idiopathic epilepsy 513

immune-mediated polymyositis 513

megaoesophagus 513

motor neuron disease 290

mucopolysaccharidosis 520

myasthenia gravis 513

spinal cord disease 276

German Shorthaired Pointer

GM2 gangliosidosis 520 steroid-responsive meningitis-arteritis 513

X-linked muscular dystrophy 513

German Wirehaired Pointer, steroid-responsive meningitis 55

Giant axonal neuropathy 512

Giant Schnauzer, methylmalonic aciduria 520

Glasgow coma scale 141, 397

Glioma 152, 153, 301, 370

Globoid cell leucodystrophy 241, 518, 519, 520, 521

Glossopharyngeal nerve (CN IX) 16, 26

Glucocerebrosidosis 241, 521

Glucocorticoids 156 (see also Corticosteroids)

Glutamate receptors 121

Glycogen metabolism defects 360

Glycogen storage diseases 519

Glycogenosis 518, 520

GM1 gangliosidosis $518,519,520,521$

GM2 gangliosidosis 39, 517, 518, 520

Golden Retriever

eosinophilic pleocytosis 55

hypomyelinating polyneuropathy 513

idiopathic epilepsy 513

myasthenia gravis 513

sensory ataxic neuropathy 291

X-linked muscular dystrophy 508, 513

Gordon Setter, cerebellar cortical degeneration 513

Grand mal seizures 119

Granulomatous meningoencephalomyelitis 57,210-11, $241,256,266,275,299,301,370$

(see also GM1 gangliosidosis; GM2 gangliosidosis)

Great Dane

core-like myopathy 513

distal sensorimotor polyneuropathy 290,513

megaoesophagus 513

myotonic myopathy 358,513

orthostatic tremor 513

Wobbler syndrome 513

Great Dane/Bloodhound cross, motor neuron disease 290

Great Dane/St Bernard cross, motor neuron disease 290

Grey matter 28, 29

Greyhound

megaoesophagus 513

neuropathy 513

steroid-responsive meningitis-arteritis 513

Griffon Briquet, motor neuron disease 290

H-reflex 65, 66

Haemangiosarcoma 299

Haematology 36-9

Haematomas 402

Haemophilus influenzae 455

Haemorrhage 165

CSF analysis $50,52-3$

epidural 275, 301

spinal 256, 270, 288-9, 327

spinal cord 301

Haemorrhagic parenchymal contusions 403

Haemostasis 42-3

Halothane 416

Harrier Hound, spinal cord disease 276

Haw's syndrome 193

Head

bobbing $8,238,247$

neurological abnormalities 213-31

pressing 4,5 
Head continued

lesion localization 27

tilt 5, 195-212, 216

central vestibular diseases 203-12

clinical signs 195

differential diagnosis 197-8

lesion localization 27, 195-6

neurodiagnostic investigation 198-9

pathophysiology 196-7

trauma

peripheral vestibular diseases 199-202

aetiology 394

pathophysiology 394-5

patient assessment 395-9

treatment 399-403

and vestibular disease 198, 212

tremors 238-9

turn 5,27

Heart rate 396

Heart rhythm 396

Heat therapy 488, 490-1

Hemidilated pupils 184

Hemifacial spasm 192

Hemilaminectomy 435, 440

Hemi-neglect syndrome 4

lesion localization 27

Hemiparesis 7

lesion localization 28, 30

Hemiplegia 7

lesion localization 28,30

Hemivertebrae 79, 80, 312

breed associations 510, 512515

Hemi-walking 18

Hepatic encephalopathy 148-9, 245

Hepatocerebellar disease 510

Hepatoencephalopathy 36

Hepatozoon americanum 363

Hepatozoon canis 363

Herbal medicines 500

Hereditary diseases see Genetic diseases

Hereditary myelopathy, breed associations 514

Hereditary myopathy, breed associations 509

Herpesvirus 158

Hetastarch 399

Himalayan cat

abnormal visual pathways 187,188

hyperchylomicronaemia 291

Hippus 181

Histological stains $97-8$

Histoplasma capsulatum 45

Histoplasmosis 158

History taking 1-4

Hopping test 18

Horner's syndrome 181-3, 219, 328

lesion localization 30

pharmacological testing 174

vestibular disease 196

Husky, GM1 gangliosidosis 520

Hydrocephalus

breed associations 510, 511, 515

clinical signs 145-6

congenital 188

diagnosis $146-7$

and eye disease 179-80

surgical management 147-8

Hydrotherapy 492
Hydroxycarbamide 467

Hyperadrenocorticism 36, 42, 290, 292

Hyperaesthesia 202, 252

lesion localization 27, 28, 30, 31, 33

Hyperbaric oxygen therapy 401

Hypercalcaemia 37, 344, 367

Hypercholesterolaemia 42

Hyperchylomicronaemia 299

and neuropathy in cats 38

Hypercortisolaemia 36

Hyperglycaemia 37

Hyperkalaemia 37, 344, 367

Hypermagnesaemia 37

Hypermetria 7

Hypernatraemia 37, 150, 245, 344

Hyperosmolality 37

Hyperoxaluric peripheral neuropathy 38

Hyperpathia 252

Hyperphosphataemia 40, 367

Hypertension 177

Hypertensive encephalopathy 165-6

Hyperthermia 422

Hyperthyroid myopathy in cats $354-5$

Hyperthyroidism 37, 42, 290

Hypertrophic neuropathy 517

Hypertrophic osteodystrophy 271

Hyperventilation 413

Hyperviscosity syndrome 38

Hypervitaminosis A 256, 264, 301, 320

Hypervitaminosis D 344

Hypoadrenocorticism 36, 42, 227, 290, 344

Hypoaesthesia 27, 31, 252

Hypoalbuminaemia 367

Hypoalgesia, facial 13, 22, 27

Hypocalcaemia 37, 40, 245, 344, 367

Hypoglossal nerve (CN XII) 16, 26

Hypoglycaemia 37, 42, 149-50, 245, 271

Hypokalaemia 37, 344, 508

Hypokalaemic myopathy $360-1$

Hypomagnesaemia 37

Hypometria 7

Hypomyelination 244, 513, 516, 517

Hyponatraemia 37, 150, 245, 344

Hypo-osmolality 37

Hypophosphataemia 37

Hypotension 271, 417, 430

Hypothalamus 25

olfaction 27

Hypothermia 401, 422

Hypothyroid myopathy 354

Hypothyroidism 37, 42, 199, 204, 290, 292 and CNS dysfunction 151

Hypoventilation 422, 486

Hypovolaemia 367

Hypoxaemia 271, 422

Hypoxia 37

Idiopathic epilepsy 117

breed associations 509, 510, 512, 513, 514, 515, 516,517

Idiopathic internal ophthalmoplegia 183

Idiopathic laryngeal paralysis 510, 511

Idiopathic polymyositis 363-4

Idiopathic tremors 514, 517

Imipenem 456

Imipramine 386

Immune myositis 38 
Immunosuppressants $462-5$

Inborn errors of metabolism

lysosomal storage diseases 109

ophthalmic manifestations 176

organic acidurias 108-9

Infarction 299

Infectious diseases 34, 158-9, 177 (see also specific diseases and agents)

Infectious meningitis 256, 264

Infectious meningoencephalomyelitis 38, 275, 301, 370

Inherited junctional disease 290

Inherited myopathies 290, 358

Inherited peripheral neuropathies 290

Inner ear

anatomy 197

trauma 202

tumours 199-200

Inspiratory stridor 219

Insulinoma 38, 42, 149, 292, 301

Intention tremor 233

(see also Tremor)

Interferential electrical stimulation 491

Intermittent positive pressure ventilation (IPPV) 416, 420, 423-5

Intervertebral disc disease $256,256,275,299,301$, 370,374

feline 310

type I 303-9

type II 309-10

Intra-arachnoid cysts $197,203,256,275,284-5,299$, 301, 315-16

Intracerebral steal syndrome 410

Intracranial disease

anaesthetic considerations $411-18$

analgesia 417

supportive care 416

Intracranial hypertension 403

Intracranial pressure $185,396,398,411,412-13$

Intracranial structures 25, 26

Intraventricular shunt placement 448-9

Involuntary movements 8-9 (see also Tremor)

Irish Setter

cerebellar dysplasia 514

globoid cell leucodystrophy 520

idiopathic epilepsy 514

lissencephaly 514

megaoesophagus 514

sensory neuropathy 290

Irish Terrier, muscular dystrophy 514

Irish Wolfhound, startle disease 249

Ischaemia 164, 389

Isoflurane 415

Italian Spinone

cerebellar degeneration 514

laryngeal paralysis polyneuropathy complex 290

Itraconazole 268, 458-9, 469

Ivermectin

adverse reactions 468

toxicity $160-1$

Jack Russell (Parson Russell) Terrier congenital myasthenia gravis 514 hereditary ataxia 238, 514

Japanese Spaniel (Chin), GM2 gangliosidosis 520

Japanese Spitz, muscular dystrophy 514

Jaw tone 12

Jerk nystagmus 15, 195
Keeshond, epilepsy 119, 514

Keratoconjunctivitis sicca 14, 216

Kerry Blue Terrier, cerebellar cortical degeneration, 514

Ketamine 407, 414, 415, 419, 429

Ketoconazole 211, 458

Klebsiella pneumoniae 455

Kooiker Hound, hereditary necrotizing myelopathy 514 spinal cord disease 276

Korat cat GM2 gangliosidosis 518 lissencephaly 508

Kyphosis 6

Labrador Retriever axonopathy 514 cerebellar cortical degeneration 514

distal sensorimotor polyneuropathy 290

exercise-induced collapse 365,514

idiopathic epilepsy 514

laryngeal paralysis polyneuropathy complex 290

mucopolysaccharidosis 520

muscle stiffness 249

myopathy 514

narcolepsy-cataplexy 514

reflex myoclonus 248, 514

spinal cord disease 276

spongiform degeneration 143

Lacosamide $125,126,133,407-8$

Lacrimal gland function 173

Lactate 43, 346-7

Lafora disease 519,521

Lameness 8

Laminectomy 435, 437-9, 441

Lamotrigine 126, 132

Laryngeal paralysis-polyneuropathy complex 512 , 515,516

Laryngeal paresis/paralysis 222, 226-7 breed associations 516 lesion localization 27

Laser therapy 493

Lateral cutaneous sural nerve 331

Lateral funiculus 29

Lateral geniculate nucleus 27

Lateral thoracic nerve 329, 331

Lead toxicity 39,160

Leflunomide 465

Leishmania spp. 45, 457

Leonberger laryngeal paralysis polyneuropathy complex 290 polyneuropathy 514

Leptospira

australis 362 icterohemorrhagiae 362

Lesion localization

altered consciousness 138-40

brainstem 27-8

cerebellum 28

extracranial structures 25

forebrain 27 head tilt 195-6

head/face abnormalities 221-2

intracranial structures 25, 26

muscle 33

neuromuscular junction 32-3

nystagmus 195-6

peripheral nerves $31-2$

spinal cord 28-31

Leucodystrophy 512 
Leucoencephalomyelopathy 515

Levetiracetam 125, 126, 128, 132, 133, 134, 406-7

Lhasa Apso

hydrocephalus 145

lissencephaly 514

Lidocaine 415, 429

Limber tail $370,380-1$

Limbic system 27, 140

Lip

paralysis 27

pinch

Lipid storage myopathy 360

Lipoma 370, 377

Lissencephaly 180, 508, 514, 516517

Lomustine 211, 466, 469

Longhaired Dachshund, sensory neuropathy 290

Lordosis 5

Losigamone 126

Lower motor neurons 29, 30

Lumbosacral fracture/luxation 370

Lumbosacral intumescence 25

Lumbosacral plexus trauma 339

Lumbosacral spinal disease 237

Lumbosacral trauma 339

Lumbosacral vertebral column surgery 443-4 dorsal laminectomy 443

foramenotomy/facetectomy 443

lumbosacral vertebral column stabilization 444

Luxury perfusion 410

Lyme disease 45

Lymphocytic pleocytosis 54

Lymphoma 37, 336, 370 spinal $319-20$

Lymphoreticular tumours 299 radiation therapy 478

Lysosomal storage disease $36,39,42,109,197,203$, 243-4, 299

Magnetic resonance imaging 87-91, 175, 332, 421

Maine Coon, spinal muscular atrophy 508

Malignant hyperthermia 366-7

Malonic aciduria 520

Maltese

generalized tremor syndrome 245, 514

hydrocephalus 145

malonic aciduria 520

necrotizing meningoencephalitis 54, 514

Mammary gland carcinoma 299

Mannosidosis 240, 518

Manx cat, sacrocaudal dysgenesis 378,508

Massage 491

Masseter muscle atrophy 13

Masticatory muscle atrophy 215-6, 222, 228-9

Masticatory myositis 228

Maximum motor nerve conduction velocity $62-3$

Maximum sensory nerve conduction velocity 65

Medetomidine 414, 418, 419, 429

Medial cutaneous antebrachial nerve 331

Medial geniculate nucleus 27

Median nerve 329, 331

Medulla oblongata 25, 26, 27

Medullary reticular formation 28

Megaoesophagus 227-8, 222

breed associations 513, 514, 515, 516, 517

lesion localization 27

Meloxicam 429

Menace response 9,10,168, 169, 170
Meningioma 152, 153, 299, 301, 370

radiation therapy $474-5$

spinal 317

Meningitis 158

Meningitis/myelitis 299

Meningocele 313, 376

Meningoencephalitis 198, 205

Meningoencephalomyelitis 286

Meningomyelitis 256, 264, 336

Meningomyelocele 313

Mental status 136

brainstem lesions 28

cerebellar lesions 28

forebrain lesions 27

paraparesis 297

peripheral nerve lesions 32

spinal cord lesions $30-1$

(see also Consciousness)

Meropenem 469

Merosin deficiency 356-7

Mesencephalon see Midbrain

Metabolic acidosis 344

Metabolic diseases 34, 148-51, 176, 180 breed associations, 517-21

Metabolic myopathies 290

Metabolic response to injury 488

Metaldehyde toxicity 39

Metencephalon see Pons dorsal see Cerebellum

Methadone 413, 419, 428

Methotrexate 467

Methylmalonic aciduria 108, 519, 520, 521

Methylprednisolone 469

Metronidazole 456-7, 469 toxicity $198,212,243$

Microalbuminurea 44

Microbiology 44-5

Microprotein in CSF $50,51,53-4$

Micturition

dysfunction

aetiology 384-6

diagnosis $383-4$

lesion localization 384

prognosis 387

supportive care $482-4$

treatment $386-7$

neuroanatomy 381

phases $382-3$

Midazolam 414, 415, 419, 429

Midbrain 25, 26, 27

Middle ear

anatomy 197

trauma 202

tumours 199-200

Miniature Pinscher generalized tremor syndrome 245 mucopolysaccharidosis 520

Miniature Poodle, spinal cord disease 276

Miniature Schnauzer

ceroid lipofuscinosis 520

megaoesophagus 515

mucopolysaccharidosis 520

muscular dystrophy 515

myotonia congenita 515

Miniature Wirehaired Dachshund, Lafora disease 250

Miosis 167 181, 182

Mitochondrial myopathy 359, 360 
Molecular testing 45, 158

Mononeuropathy 328

Monoparesis

clinical signs 328-9

definition 7

differential diagnosis 331-2

diseases causing $332-41$

lesion localization 30-1, 329-30

neurodiagnostic investigation 332

pathophysiology 330-1

Monoplegia 7, 328

Morphine 428

Motor neuron disease see Spinal muscular atrophy

Motor tracts 29

Motor unit 33

MRI see Magnetic resonance imaging

Mucolipidosis II 518

Mucopolysaccharidosis 39, 240, 299, 301, 311, 518, $519,520,521$

Multiple cartilaginous exostoses 82

Multisystem neuronal degeneration 511, 516

Muscle

anatomy and function 33

biopsy $94-8,348$

clinical signs of dysfunction 33

cramps 233

injury, markers $43-4$

lesions 33

stiffness, potassium-aggravated 361

ultrastructure 95-7

Muscle mass abnormalities

cerebellar lesions 28

muscle lesions 33

spinal cord lesions $30-1$

Muscle tone abnormalities

brainstem lesions 28

cerebellar lesions 28

forebrain lesions 27

muscle lesions 33

peripheral nerve lesions 32

spinal cord lesions $30-1$

Muscular dystrophy $38,355-7$

breed associations $508,509,513,514,515,516$

Musculocutaneous nerve 329, 331

Myasthenia gravis 38, 64, 227, 229, 290

breed associations $508,509,513$

congenital 290, 351-2, 512, 514, 516

immune-mediated 349-51

Mycophenolate 462-3

Mydriasis 167, 181

Myelencephalon see Medulla oblongata

Myelin 100

Myelitis 336

Myelography 83-5, 332, 370, 421

Myelopathy 509

Myoclonus 8, 232, 233, 236, 237-9

(see also Reflex myoclonus)

Myofibre adenosine triphosphatase 97

Myoglobinuria 44, 347

Myokymia 8, 233, 234, 236, 238

Myopathies $38,352-67$

breed associations 508, 510, 512, 513, 514, 516

(see also specific diseases)

Myositis 38

Myotonia 8, 236

Myotonia congenita $361-2,511,515$

Myringotomy
Narcolepsy 137, 160, 250

forebrain lesions 27

lesion localization 27

Narcolepsy-cataplexy 250

breed associations, 512, 514, 515,

Nasopharyngeal polyps 198, 201

Neck and back pain

clinical signs 252

differential diagnosis $256-70$

lesion localization 252-3, 254

neurodiagnostic investigation 256

pathophysiology 254-5

Necrotizing encephalopathy 509,517

Necrotizing leucoencephalitis 517

Necrotizing meningoencephalomyelitis 211-12 breed associations 511, 514, 515

Necrotizing myopathies 364

Neisseria meningitides 455

Neospora caninum 45, 107, 363, 460

Neosporosis 158, 209

Nephroblastoma 299, 301, 318-19

Nerve biopsy 348

Nerve conduction velocity motor 62-3 sensory 65

Nerve root signature $8,253,328,332$

Nerve sheath tumours 299, 301, 334-6, 370

Neurapraxia 330

Neuroaxonal dystrophy 241, 299 breed associations 508, 511, 515, 516

Neurodegenerative diseases 110, 143-4,197, 203

Neuroendoscopy 447

Neuroepithelioma 301

Neurogenic intermittent claudication 332, 371

Neurogenic reflexes 410

Neuroimaging

computed tomography $85-7$

magnetic resonance imaging $87-91$

myelography 83-85

radiography $59-64$

ultrasonography 91-2

Neurological examination

aims 1

general physical examination 4

hands-off

abnormal involuntary movements 8-9

consciousness/awareness/behaviour 4

gait $6-8$

posture and body position 5-6

hands-on

cranial nerve assessment $9-16$

postural reaction testing 17-18

sensory evaluation $22-4$

spinal reflexes 19-22

history 1-4

lesion localization 19, 26

Neuromuscular disease 238 paraparesis 298-9

Neuromuscular electrical stimulation $492-3$

Neuromuscular junction 344

anatomy and function 32

clinical signs of dysfunction 32-3

Neuromuscular transmission disorders $349-52$ botulism 227, 290

congenital myasthenia gravis $351-2$

drug toxicity 352

immune-mediated myasthenia gravis 349-51 organophosphate and carbamate toxicity 352 
Neuromyotonia 233, 238, 247

Neuronal degeneration 511

Neuro-ophthalmic syndromes 175-94

Neuro-ophthalmological assessment

cerebrospinal fluid analysis 175

chromatic papillary light reflex 170

dazzle reflex 171

electroretinography 174, 175

extraocular muscles 171

eyeball position and movement 171-2

lacrimal gland function 173

magnetic resonance imaging 175

motor control of eyelids 173

pupil function 174

pupillary light reflex 9, 27, 169, 170

somatosensory innervation 172

swinging flashlight test 170-171

sympathetic innervation 173

vision 168, 169

visual evoked potentials 175

Neurosurgery

common procedures 433

considerations prior to surgery 433-4

cranial surgery $444-50$

emergencies $432-3$

indications $432-3$

postoperative care 435

spinal surgery $435-44$

Neurotmesis 331

Neutrophilic pleocytosis 55

New Zealand Huntaway Dog, mucopolysaccharidosis 520

Newfoundland

distal sensorimotor polyneuropathy 290

immune-mediated polymyositis 515

megaoesophagus 515

Nictitating membrane see Third eyelid

Niemann-Pick disease 241, 291, 517, 518, 519

Nissl substance 106

Nitrosoureas 466

Nitrous oxide 416

Nociception 23-24, 254, 298

Non-steroidal anti-inflammatory drugs 461

(see also specific drugs)

Norwegian Forest cat, glycogenosis type IV 291, 518

Nostril paralysis 27

Nutritional myopathy 352-3

Nutritional support $400-1,488$

Nystagmus 167

central vestibular diseases 203-12

clinical signs 195

congenital 187

differential diagnosis 197-8

lesion localization 27, 195-6

neurodiagnostic investigation 198-9

pathophysiology 196-7

pendular 187, 195

peripheral vestibular diseases 199-202

physiological 15, 172

positional

spontaneous 189

Obturator nerve 330,331

Occipital lobe 26

Ocular tremor 189

Oculomotor nerve (CN III) 10-11, 26

neoplasia 183

Oesophagitis 227
Old English Sheepdog

cerebellar cortical degeneration, 515

cytochrome oxidase deficiency 520

muscular dystrophy 515

Olfaction 27

Olfactory nerve (CN I) 9, 26, 27

Oligodendroglioma 299

Ophthalmological electrophysiological testing 72

Ophthalmoplegia 167, 183

Opioids 413, 419 (see also specific drugs)

Opisthotonus 28, 137

Opsoclonus 167

Optic chiasm 26 lesions 179

Optic disc atrophy $177-8$

Optic disc hypoplasia 177

Optic nerve (CN II) 9-10, 26

Optic neuritis 178

Organic acid analysis 347

Organic aciduria 108-9

Organophosphate toxicity $41,184,352$

Orthostatic tremors 238, 513

Osmotic diuretics 399-40

Osteochondromatosis $82,256,275,285,301,311-12$

Osteomyelitis 256, 266-9, 275, 301, 370

Otitis externa 200

Otitis interna 198, 2001-1, 229

Otitis media 198, 200-1, 229

Ototoxicity 202

Oxbutynin chloride 386

Oxcarbazepine 126

Oxypolygelatin 399

Pacing in forebrain lesions 27

Pain management see Analgesia

Palpation 24

Palpebral reflex 9, 12, 215

Pan-ophthalmoplegia 167

Papilloedema 178

Papillon, neuroaxonal dystrophy 515

Paraesthesia 252, 328

Parainfluenza 158

Paraneoplastic neuropathy 293

Paraparesis

clinical signs 297-9

definition 7

differential diagnosis 301--2

diseases causing

anomalous 311-16

degenerative 302-11

idiopathic 322-3

inflammatory 320-2

metabolic 316

neoplastic 316-20

nutritional 320

toxic 325-7

traumatic 323-5

lesion localization 299-300

neurodiagnostic investigation 301

pathophysiology 300

Paraplegia 7

Parasitic disease 159

Paresis $7-8$

vestibular disease 196

Parietal lobe 26

Paroxysmal disorders diagnosis 237 


\section{Paroxysmal disorders continued}

familial reflex myoclonus 248

hypertonicity (hyperexplexia) 249

Lafora disease 250

lesion localization 234

'Scotty cramp' 248-9

sleep disorders 250-1

PARR 58

Parvovirus 158

Patellar reflex 21, 22

Pediculectomy 443

Pekingese divergent strabismus 188 hydrocephalus 145

Pelvic trauma 339-40

Pembroke Welsh Corgi degenerative myelopathy 515 spinal cord disease 275

Pendular nystagmus 187, 195

Penicillins 456

Pentastarch 399

Perception 138

Perineal nerve 331

Perineal reflex 22, 67

Perineurial (Tarlov) cysts 256, 263

Peripheral nervous system anatomy and function 31-2 disease anaesthesia 422-3 breed associations 509 dysfunction 32, 235-6 injury 339 nerve biopsy 98-100

Peripheral sensory neuropathy 510

Peripheral vestibular diseases 198, 199-202

Peroneal nerve 330, 331 injury 340 paralysis

Persian cat hyperchylomicronaemia 291 mannosidosis 518

Persistent right aortic arch 227

Pethidine 428

Petit mal seizures 119

Petrissage 491

P-glycoprotein 454, 468

Phenobarbital 125, 126, 127, 128, 129, 130, 133, 406, 414

Phenoxybenzamine 387

Phenylpropanolamine 386

Phenytoin 26

Physical rehabilitation active muscle contraction 489, 492 gait training 493-5 interferential electrical stimulation 489, 491 massage 488, 491 neuromuscular electrical stimulation $489,492-3$ proprioceptive training, 489, 494-5 range of motion $488,489-91$ thermotherapy $488,490-1$

Physiological nystagmus 15, 172

Physiotherapy see Physical rehabilitation

Physitis see Vertebral physitis

Pituitary gland tumours 152, 153 radiation therapy $475-8$

Placing response see Visual placing response

Pleurothotonus 5 forebrain lesions 27
Plexus neuritis 336

Plotthound, mucopolysaccharidosis 520

Pneumonia 422

Podhale Shepherd, laryngeal paralysis polyneuropathy complex 290

Polioencephalomyelopathy 246, 509

Polish Lowland Sheepdog, ceroid lipofuscinosis 520

Polyarthritis 256, 269, 271

Polymyopathy 269

Polymyositis 227, 256, 269, 271, 290, 363-4 breed associations 510, 513, 515, 517

Polyneuropathy 290, 513, 514, 515,

Polyradiculoneuritis 290, 293-4

Pomeranian, hydrocephalus 145, 515

Pons 25, 26

Poodle

globoid cell leucodystrophy 520

idiopathic epilepsy 515

narcolepsy-cataplexy 515

neonatal encephalopathy 515

Portosystemic shunt

Portuguese Water Dog, GM1 gangliosidosis 520

Postural reactions

abnormalities, lesion localization 27, 28, 30-1, 32, 33

paraparesis 298

testing $17-18$

Posture 5-6

abnormalities, lesion localization 27, 28, 30-1, 32, 33 paraparesis 297

Potassium bromide 129, 130-1, 134, 407

Pourfour du Petit syndrome 183

Prazosin 387

Prednisolone 211, 266, 469

Prednisone 211

Pregabalin 126, 132

Prehension, abnormal 27

Premedication 413, 414, 418, 422

Pressure sores see Decubital ulcers

Procarbazine 211

Progesterone 401

Progressive axonopathy 510

Propantheline bromide 386

Propofol 407, 415, 419

Proprioception 17, 27

Proprioceptive ataxia

Proprioceptive positioning 17

Proprioceptive training 489, 494-5

Protothecosis 158

Protozoal myelitis 321-2

Protozoal radiculoneuritis 294-5

Proximal sciatic nerve injuries 340

Pseudomyotonia 62

Pseudorabies 158

Ptosis 11, 167, 181, 182, 184

Pudendal nerve 331, 368, 371

Pug encephalitis 54

hemivertebrae 515

hydrocephalus 145

necrotizing meningoencephalitis 54

necrotizing meningoencephalomyelitis 515

Pulmonary atelectasis 487

Pulse oximetry 396, 425-6

Pupil

abnormalities $175,181-6$

function 174

size 11

Pupillary changes in altered consciousness 137 
Pupillary light reflex 9,27, 167, 169, 170

Pupillotonia 185

Pyramidal system 27

Pyrenean Mountain Dog, laryngeal paralysispolyneuropathy complex 290, 515

Pyriform lobe 26

Pyrimethamine 460, 469

Pyruvate 346-7

Pyruvate dehydrogenase deficiency 521

Rabies 45, 158-9, 160

Rachischisis 313

Radial nerve 329, 331 injury 339

Radiation therapy brain tumours 156-7 intracranial mass $472-4$ meningioma $474-5$

lymphoreticular tumours 478

molecular targeting and dose painting 479

palliative radiation of CNS 478-9

pituitary gland tumours $475-8$

proton radiotherapy 480

radiation dose/radiobiology $470-2$

spinal cord tumours 478

stereotactic dosing 479

Radiography 77-83

Radiosurgery, stereotactic 450-1

Range of motion 488, 489-90 passive

Reflex myoclonus 248, 514

Reflexes

biceps brachii 20

corneal 9,10

cranial tibial 21-22

cutaneous trunci (panniculus) 23-4

extensor carpi radialis 20

gag 9

gastrocnemius 21-22

palpebral 9, 12

patellar 21, 22

perineal 22, 67

pupillary light 9,27

spinal $19-22$

swallowing 16

triceps 20

vestibulo-ocular 9

withdrawal (flexor) 19-20

Regurgitation 217-19

Rehabilitation

patient assessment $481-2$

physical therapy

active muscle contraction 489, 492

cryotherapy 488,490

gait training 493-4

heat therapy $488,490-1$

interferential electrical stimulation 489, 491

massage 488, 491

neuromuscular electrical stimulation 489 ,

492-3

proprioceptive neuromuscular training 489 ,

494, 495

range of motion $488,489-91$

thermotherapy 490-1

supportive care $482-8$

treatment plan 482

REM sleep disorders 251

Remacemide 26
Remifentanil 413, 419, 429

Renal failure 37

Repetitive stimulation 64

Respiration abnormalities, lesion localization 28, 30, 31 regulation 28

Respiratory rate 396

Respiratory rhythm 396

Respiratory system care aspiration pneumonia 486-7

hypoventilation 486

pulmonary atelectasis 487

Resting anisocoria 181

Retigabine 126

Retrobulbar swelling/trauma 190, 191

Rhabdomyolysis 366-7

Rhodesian Ridgeback cerebellar abiotrophy 515 spinal cord disease 276

Riboflavin 360

Rickettsia rickettsii 45

Rigidity 236, 298

Robin Hood phenomenon 410

Rocky Mountain spotted fever 45, 55, 158

Rolling 27

Rostrotentorial craniotomy 444, 446

Rottweiler

distal myopathy 516

distal sensorimotor polyneuropathy 290

laryngeal paralysis-polyneuropathy complex 290 516

leucoencephalomyelopathy 515

motor neuron disease 290, 515

mucopolysaccharidosis 521

muscular dystrophy 515

neuroaxonal dystrophy 516

progressive polyneuropathy 515

spinal cord disease 276

spinal cyst 516

spongiosis of grey matter 515

Round cell tumours 301,370

Rufinamide 125, 126, 133

Russian Black Terrier, laryngeal paralysis polyneuropathy complex 290

Saccade 167

Sacral fractures $370,379,380$

Sacrocaudal dysgenesis 312, 376, 508

Sacrocaudal luxation 370

Sacrococcygeal fracture/luxation 380

Saluki

motor neuron disease 290

spongiosis of grey matter 516

Samoyed

cerebellar cortical abiotrophy 516

hypomyelination 516

lissencephaly 516

muscular dystrophy 516

spongiform degeneration 143

Saphenous nerve 331

Sarcoglycan deficiency 356

Schiff-Sherrington posture 6, 298 lesion localization 31

Schipperke, mucopolysaccharidosis 521

Schirmer tear test 14

Schwann cells 100

Sciatic nerve 330, 331 neuropathy 
Scoliosis 5, 256, 263 lesion localization 30

Scottish Terrier cerebellar cortical degeneration 516 'Scotty cramp' 248-9, 516

Screening for genetic disease 113-14

Sedation 413, 414, 418, 422

Seizures 8, 232-3

clinical signs 117-20

differential diagnosis 122-3

forebrain lesions 27

in hypernatraemia 150

in hyponatraemia 150

lesion localization 27, 120

neurodiagnostic investigation 123-4

pathophysiology 120-2

prophylaxis 400

terminology 118, 119

treatment emergencies 134-5, 403-8

goals $127-7$

protocols cats 133-4 dogs 127-133

strategy 124-6

(see also Status epilepticus)

Seletracetam 126

Self-mutilation 486

lesion localization 32

Senile tremor 237

Sensation abnormalities

brainstem lesions 28

cerebellar lesions 28

forebrain lesions 27

muscle lesions 33

peripheral nerve lesions 32

spinal cord lesions 30,31

Sensory evaluation 22-4

cutaneous sensory testing 22-3

cutaneous trunci (panniculus) reflex 23

nociception testing 23-4

palpation 24

Sensory neuropathy 512

Sensory tracts 29

Serum protein electrophoresis 44

Setting sun sign 146, 142

Sevoflurane 415

Shar Pei

megaoesophagus 516

methylmalonic aciduria 521

Shetland Sheepdog

dermatomyositis 516

dysmelination 516

familial epilepsy 120

Siamese cat

abnormal visual pathways 187, 188

cerebellar abiotrophy 509

ceroid lipofuscinosis 518

congenital vestibular disease and deafness 509

GM1 gangliosidosis 518

hyperchylomicronaemia 291

mucopolysaccharidosis 518

Niemann-Pick disease 291, 518

Siberian Husky

degenerative myelopathy 516

GM1 gangliosidosis 521

laryngeal paralysis 516

spinal cord disease 276
Silky Terrier

glucocerebrosidosis 521

spongiform degeneration 143

Single fibre EMG $64-5$

Skin care 384-6

decubital ulcers 385-6

self-mutilation 386

Skull fractures 402

Sleep disorders 250-1 (see also Narcolepsy and Cataplexy)

Sleep regulation 27

Sliding paper test 18

Smooth-haired Fox Terrier congenital myasthenia gravis 516 congenital vestibular disease 516

Somatosensory evoked potentials $67-8$

Spasticity 298

Special sensory systems 60

Sphynx cat, muscular dystrophy 509

Spina bifida 80, 312, 370, 375, 508, 512

Spina bifida occulta 312

Spinal cord

anatomy and function $25,28-30$

anomalies $263,275,285,301,370$

clinical signs of dysfunction 30-1

diseases, and tetraparesis 275-90

dysfunction 235-6

haemorrhage 301

injury $287,301,388-94$

malformations 299, 312-15

regions 19

segmentation 29-30

tumours 264, 285, 316-20

radiation therapy 478

(see also specific diseases)

Spinal curvature 5-6

Spinal dysraphism 299, 313

Spinal empyema 285-6, 301, 320

Spinal extrasynovial cyst 299

Spinal fracture 81, 270, 323-5

Spinal haemorrhage $256,270,288-9,327$

Spinal hyperaesthesia, paraparesis 297

Spinal luxation 270

Spinal lymphoma 319-20

Spinal malformations $79-80$

Spinal meningiomas 317

Spinal muscular atrophy 508, 510, 512, 515516

Spinal neoplasia $80-1$ (see also specific tumours)

Spinal neuroepithelioma 301

Spinal radiography $77-82$

Spinal reflexes 19-22

abnormalities 27-33

lower motor neuron 19

paraparesis 298

pelvic $20-22$

tail and anus 22

thoracic 19-20

upper motor neuron 19

(see also Reflexes)

Spinal shock 300

Spinal stabilization 435, 439-40, 441-2

Spinal stenosis 299, 312, 315

Spinal surgery

cervical spine $436-40$

general complications 435-6

indications 435

lumbosacral spine 443-4

thoracolumbar spine $440-3$ 
Spinal subarachnoid cyst 516

Spinal synovial cysts $257,275,310-11$

Spinal ultrasonography $91-2$

Spondylosis deformans 256, 257-8, 299, 301, 310, 370

Spongiform encephalopathy 508

Spongiform leucoencephalopathy 511

Spongiosis of grey matter 511, 515, 516

St Bernard

distal sensorimotor polyneuropathy 290

idiopathic epilepsy 516

Staffordshire Bull Terrier L-2-hydroxyglutaric aciduria 521 myotonic myopathy 516

Staphyloccal protein A-horseradish peroxidase 98

Staphylococcus spp. 266, 455

Static anisocoria 184

Status epilepticus $118,403-8$

Stereotactic radiosurgery $450-1,479$

Steroid myopathy 353

Steroid-responsive meningitis 38,55

Steroid-responsive meningitis-arteritis 58, 264-5, $256,266,275,286,301,370$ breed associations 509, 510, 511, 513, 517

Strabismus 11, 167

congenital 187

divergent 186, 187, 188

intracranial lesions 191

lesion localization 27

positional

Stranguria 383

Streptococcus 266, 268, 455

pneumoniae 455

Strychnine toxicity 39

Stupor 136, 149

aetiology 141

and brain tumours 151

clinical signs 137

diagnosis 141-2

lesion localization 27, 138

Subluxations 79

Suboccipital craniotomy 444, 447

Subscapular nerve 331

Succinate dehydrogenase 98

Succinylated gelatin 399

Sudden acquired retinal degeneration 177

Superficial peroneal (fibular) nerve 331

Supportive care

behaviour 487-8

bladder and bowel management $482-4$

in intracranial disease 416

nutrition 488

respiratory system $486-7$

skin care 484-6

in spinal disease 420

Suprascapular nerve 329, 331

Surgery see Neurosurgery

Sussex Spaniel, pyruvate dehydrogenase deficiency 521

Swallowing reflex 16

Swedish Lapland Dog

glycogenosis 520

motor neuron disease 291

multisystem neuronal abiotrophy 516

Swimming as rehabilitation 489

Swinging flashlight test $1170-1$

Syncope 137

Synovial cysts 256, 281, 301
Syringohydromyelia see Syringomyelia

Syringomyelia 256, 260-3, 275, 285, 299, 313, 314, 510,511

Tachycardia 137

Tail abscessation 377

Tail, anal and bladder dysfunction clinical signs 368

disorders 370-81

lesion localization 369

neurodiagnostic investigation 370

pathophysiology 369-70

Tarlov cysts see Perineurial cysts

Tear production, abnormalities 176, 193-4

Telencephalon see Cerebrum

Temporal lobe 26

Temporalis muscle atrophy 13

Testosterone 387

Tetanus 191, 275, 286-7, 341, 422

Tetraparesis

clinical signs 271-2

definition 7

lesion localization 28, 30, 33, 272

lower motor neuron diseases 290-6

neurodiagnostic investigation 274-5

non-neurological diseases causing 271

pathophysiology 273-4

spinal cord diseases $275-90$

Tetraplegia 7, 271

lesion localization 28, 30, 33

Thalamus 25

Thermotherapy 490-1

Thiamine deficiency 158, 186, 197, 204

Thiopental 415, 419

Thiopurines 463-4

Third eyelid abnormalities 175, 193 protrusion 182

Thirst 27

Thoracolumbar disc fenestration 442

Thoracolumbar lateral corpectomy 442

Thoracolumbar vertebral column surgery dorsal laminectomy 441

foramenotomy and/or pediculectomy 443

hemilaminectomy $440-1$

thoracolumbar disc fenestration 442

thoracolumbar lateral corpectomy 442

thoracolumbar vertebral column stabilization 441-2

Thromboelastography 43

Thromboembolic disease 275, 301

Thrombosis 299

Tiagabine 126

Tibetan Mastiff, hypertrophic neuropathy 291, 517

Tibetan Terrier, ceroid lipofuscinosis 521

Tibial nerve 330, 331 injury 340

Tick paralysis $290,295-6$

Tick-borne infections 38

Todd's paralysis 118

Tongue

abnormalities 219

paralysis, lesion localization 27

Tonkinese cat, congenital vestibular disease 509

Topiramate, 125, 126, 131-2, 133

Torticollis 6

lesion localization 30

Toxoplasma gondii 45, 363, 460

Toxoplasmosis 158, 209 
Toy Poodle, hydrocephalus 145

Tramadol 429

Transfrontal craniotomy 444, 445

Transitional vertebrae $79,312,315,374$

Transsphenoidal hypophysectomy 447

Trauma 35, 160

caudal lumbar 339

disc herniation 275, 287, 299, 301

head 198, 212, 394-403

inner ear 202

lumbosacral 339

lumbosacral plexus 339

middle ear 202

pelvic 339-40

Treadmill 489

Tremor 8,

clinical signs 233

differential diagnosis, 236-7

head 238-9

lesion localization 234

limb 237-8

neurodiagnostic investigation 237

pathophysiology 234-6

Triceps reflex 20

Trigeminal nerve (CN V) 12-13, 26, 172, 214, 215, 216 palsy 229

Trimethoprim/sulphonamides 456, 469 (see also specific drugs)

Trochlear nerve 11-12, 26 lesions 186

Troponin 1346

Trypanosoma cruzi 363

Tumoral calcinosis see Calcinosis circumscripta

Ulcers

cornea 192

decubital $485-6$

Ulnar nerve 329,331

Ultrasonography $91-2$

Upper motor neurons 27, 29

Uraemic encephalopathy 37

Urethra

incompetence 385

innervation 381

obstruction 385-6

Urinalysis 384

Urinary incontinence, supportive care 382-4

Urine protein:creatinine ratio 44

Urine retention 385-6 lesion localization 30, 31

Urodynamic testing 384

Vagus nerve (CN X) 16, 26

Valproate 26

Vascular diseases 161-6, 237-8

Vasculitis 299

Ventral decompression see Ventral slot

Ventral funiculus 29

Ventral slot $435,436-7$

Ventricular system 46

Ventriculoperitoneal shunts 148

Vertebral anomalies 256, 264, 275, 285, 301, 314-15, 334,370

Vertebral body tumours $299,312,317-18$

Vertebral fractures/luxations 287-8, 299, 379

Vertebral malformations 299, 312-15

Vertebral palpation 254

Vertebral physitis 299, 301, 322, 370
Vertebral plasma cell tumours 318

Vertebral-sacrocaudal dysgenesis 370

Vestibular disease 188 bilateral, neurological examination congenital 199, 508, 509, 511, 512, 516 paradoxical 196

(see also Central vestibular disease; Peripheral vestibular diseases)

Vestibulocochlear nerve (CN VIII) 14-16, 26

Vestibulo-ocular reflex 9

Vision 27, 167-94

assessment 10, 167-75 disorders 175-94

Visual evoked potentials 175

Visual placing response 18,168

Vizsla

epilepsy 119,517

focal polymyositis 517

von Willebrand's disease 41

Wakefulness 27, 138, 139

Wallerian degeneration 100

Wandering 27

Water balance 27

Weaving

Weight shift

Weimaraner hypomyelination 517 steroid-responsive mengitis-arteritis 517

Welsh Corgi ceroid lipofuscinosis 521 mucopolysaccharidosis 521

West Highland White Terrier globoid cell leucodystrophy 290, 521 idiopathic tremors 517 spinal cord disease 276

Wheelbarrowing 18

White matter 28-9

Wirehaired Dachshund Lafora disease 521 mucopolysachharidosis 521

Wirehaired Fox Terrier cerebellar hypoplasia 517 idiopathic epilepsy 517 lissencephaly 517 megaoesophagus 517

Withdrawal (flexor) reflex pelvic limb 20 thoracic limb 19

Wobbler syndrome (cervical stenotic myelopathy) breed associations $510,512,513$ clinical signs 256-7, 275, 276 diagnosis $277-8$ pathogenesis 276-7 treatment and prognosis 278-9

Xanthochromia 50

Xeromycteria 167, 185, 193

X-linked myotubular myopathy 358

Yorkshire Terrier

hydrocephalus 145

necrotizing encephalopathy 517

necrotizing leucoencephalitis 517

necrotizing meningoencephalitis 54

Zonisamide 125, 126, 128, 132, 133 